\title{
Suggesting a Reliable Method of Calculating Charge Collection Efficiency in Charge-transfer Measurement for Brush Discharges
}

\author{
Nirmalya Basu \\ Master of Engineering (Electronics and Telecommunication Engineering) \\ Date of Birth: May 3, 1984 \\ Email: nirmalya1basu@gmail.com \\ Mobile: +919007757186 \\ Address: 162/B/185 Lake Gardens, Kolkata, West Bengal, India, PIN: 700045.
}

\begin{abstract}
Induced charge errors lead to underestimation of transferred charge in brush discharges when the measurement is done using fast-response unshielded probes. The discrepancy observed between the values of chargecollection efficiency for different charge-transfer thresholds obtained theoretically by Walmsley [2] and those obtained experimentally by Chowdhury et al [3] necessitates figuring out ways to improve the mathematical model used by Walmsley. A close perusal of the said work by Walmsley [2] has pointed out an error therein-in the use of the equation $r_{\text {eff }}=K D$. I propose here a way to get rid of this error, and in doing so, I propose a method of calculating the charge-collection efficiency in charge-transfer measurement for brush discharges more reliable than that used by Walmsley [2].
\end{abstract}

\section{Introduction}

Electrostatic discharges present us with hazardous situations in industrial processes which involve the use of insulating solids and liquids, such as, sheet processing, filling up of flexible intermediate bulk containers, transporting liquid fuels through plastic pipes etc. where flammable vapours could be present in the vicinity. In these processes, the charging of the insulating solids involved could result from tribocharging or impact of dust or even the flow of insulating liquid past the solid surface. It has been suggested that in order to prevent ignition of flammable vapours, the charge transferred in the discharge process should not exceed the following thresholds: $60 \mathrm{nC}$ for Gas Group IIA, $30 \mathrm{nC}$ for Gas Group IIB and 10 $\mathrm{nC}$ for Gas Group IIC [1]. Hence, it is necessary that components and equipment which are to be used in explosive atmospheres and are likely to acquire charge be subjected to electrostatic tests under worst-case conditions to determine if these could result in discharges in which the transferred charge might exceed the threshold limits sated above.

However, as has been shown theoretically by Walmsley [2] and experimentally by Chowdhury et al [3], induced charge errors in measurement of transferred charge in brush discharges using fast-response, unshielded probes could lead to erroneous classification of unsafe equipment as safe. Walmsley calculated the charge-collection efficiency (ratio of the charge transferred in the external measurement circuit to that transferred across the discharge gap) to be $56 \%$ for $60 \mathrm{nC}$ transfer and about $20 \%$ for $30 \mathrm{nC}$ and $10 \mathrm{nC}$ transfers [2]. Experiments by Chowdhury et al [3] have shown the corresponding values to be $70 \%$ for 60 $\mathrm{nC}$ charge transfer and more than $50 \%$ for $10 \mathrm{nC}$ charge transfer. 
We find that there is significant discrepancy between the values of charge-collection efficiency found theoretically [2] and those found by experimental means [3]. This calls for figuring out ways of improving the mathematical model used by Walmsley so that the results obtained thereof might be closer to the experimental results. In this work, we point out an error in the aforementioned mathematical model and suggest a solution such as would help us get rid of this error. The solution is expected to do away with the discrepancy between theory and experiment.

\section{Review of Literature}

\subsection{Walmsley's Model and the Probable Source of Error}

In this model [2], an earthed sphere of radius $a$ has been considered to be so situated that its centre lies on the line passing perpendicularly through the centre of a thin insulating disk of radius $R$, uniformly charged with a charge density $\sigma$. The separation between the centre of the disk and that of the sphere is $D$. This is depicted in Fig. 1 (reproduced from [2]).

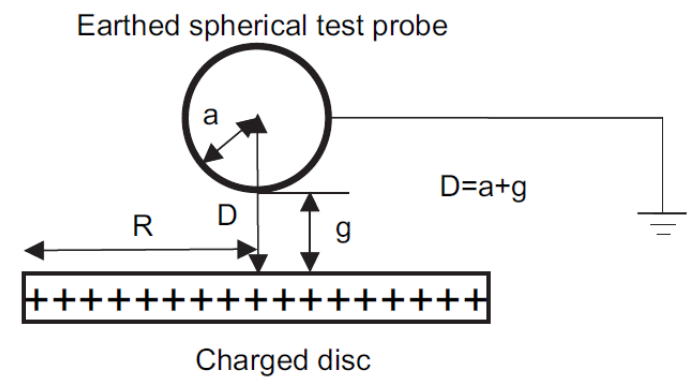

Fig. 1. Model of the Disk-sphere Discharge System (reproduced from [2])

It has been assumed that from a central patch of radius $r_{\mathrm{d}}$ on the disk, a mean fraction $\alpha_{\mathrm{d}}$ of the initial charge is removed by the discharge. This gives an effective discharge radius $r_{\text {eff }}$ given by $r_{\mathrm{eff}}^{2}=\alpha_{\mathrm{d}} r_{\mathrm{d}}^{2}$ [2]. It may be noted that $r_{\mathrm{eff}}$ is the radius of that hypothetical circular area on the disk from which the entire charge is assumed to have been removed, this quantity of charge being equal to the charge actually removed by the discharge, so that we have $\Delta Q_{\mathrm{g}}=$ $\pi r_{\text {eff }}^{2} \sigma$, where $\Delta Q_{\mathrm{g}}$ is charge transferred across the gap. It has also been assumed that

$$
r_{\text {eff }}=K D \ldots(1) \text {, }
$$

where $K$ is a constant [2].

This assumption, $r_{\text {eff }}=K D$, along with the relationship $\Delta Q_{\mathrm{g}}=\pi r_{\text {eff }}^{2} \sigma$ leads us to the relationship

$$
\Delta Q_{\mathrm{g}}{ }^{\prime}=\sigma^{\prime} K^{2} D^{\prime 2} \ldots(2),
$$

where the primed symbols represent dimensionless quantities [2].

Equation (2) implies 


$$
D^{\prime 2}=\frac{\Delta Q_{g}^{\prime}}{K^{2} \sigma^{\prime}} \quad \ldots(3)
$$

Equation (3) shows an inverse relationship between $D^{\prime}$ and $\sigma^{\prime}$, which implies that as $\sigma^{\prime}$ increases, $D^{\prime}$ decreases, and vice-versa. This is contrary to our understanding of the discharge process. This is because as $\sigma^{\prime}$ increases, the magnitude of the electric field in the discharge gap increases thereby resulting in the occurrence of the discharge at a greater sphere-disk separation. Theoretical and experimental evidences from the available literature in favour of this argument are given in section 2.2. Since equation (3) results from the relation $\Delta Q_{\mathrm{g}}=\pi$ $r_{\mathrm{eff}}^{2} \sigma$ which is valid and the empirical relation (1), we suspect the validity of equation (1). Thus, one source of error in Walmsley's model seems to be the use of equation (1).

\subsection{Supportive Evidences for Our Argument from Existing Literature}

Heidelberg [4] has shown that the electric field at the surface of the earthed sphere in the disk-sphere system shown in Fig. 1 is given by

$$
E_{s}=-\frac{\sigma}{2 \varepsilon}\left\{3-\frac{D}{a}+\frac{(D-a)\left(\frac{D}{a}-3\right)+\frac{R^{2}}{a}}{\sqrt{(D-a)^{2}+R^{2}}}\right\} \ldots
$$

This equation shows that as the charge density on the disk increases, the magnitude of the electric field on the surface of the earthed sphere also increases. Since Landers criterion for the breakdown field (equation (5)) [5] has been used by Walmsley [2] in his calculations, it is evident that the breakdown field has been assumed to depend only on the sphere radius $a$.

$$
E_{0}=2.28\left(1+\frac{1}{\sqrt[3]{100 a}}\right) \quad \mathrm{MV} / \mathrm{m} \ldots
$$

Thus, for a particular sphere radius, the breakdown electric field is assumed to remain constant. Hence, it is reasonable to expect that for a given sphere radius, if the charge density on the disk is higher, the electric field at the sphere surface reaches the breakdown value at a greater disk-sphere separation, i.e., the discharge ensues at a greater disk-sphere separation. This argument is supported by calculations due to Walmsley [2] and Heidelberg [4] and experimental results obtained by Fredholm et al [6].

Walmsley [2] has calculated the values of disk-charge density required for breakdown of the discharge gap for different values of $D^{\prime}$ and $R^{\prime}$ (the dimensionless quantities corresponding to $D$ and $R$ ). The results are shown in plots in Fig. 2. 


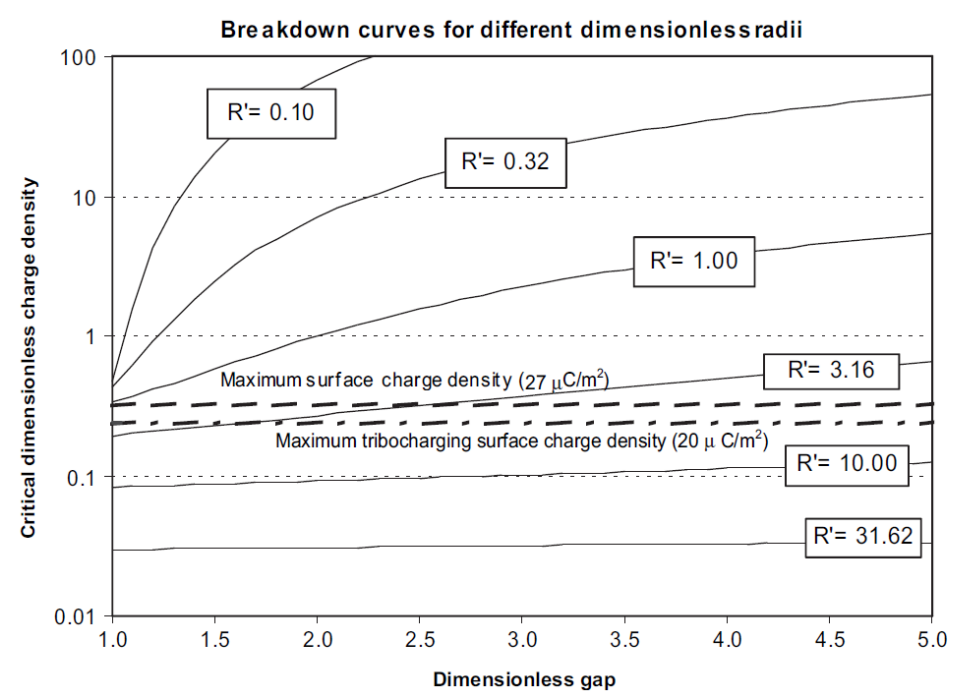

Fig. 2. Breakdown Criterion Curves (Reproduced from [2])

From Fig. 2, it can be observed that as the charge density increases, the disk-sphere separation at which discharge occurs also increases.

Heidelberg [4] has plotted the disk-sphere separation $D$ at which the breakdown field strength is reached as a function of disk radius $R$ for different values of disk-charge density $\sigma$ using equation (4). He has used the value $45 \mathrm{kV} / \mathrm{cm}$ as the breakdown electric field strength for his calculations. The plots are shown in Fig. 3.

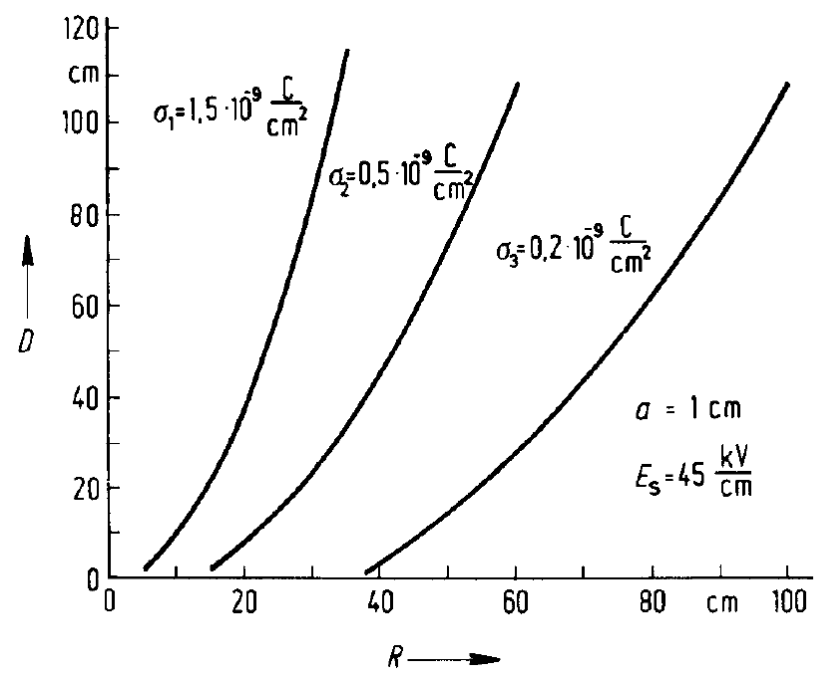

Fig. 3. Distance $D$ at Which the Breakdown Field Strength is reached for the System According to Fig. 1, as a Function of $R$ (Reproduced from [4])

These graphs (Fig. 3) also show that for a specific value of $R$, as the value of $\sigma$ increases, that of $D$ also increases. 
Fredholm et al [6] has experimented with brush discharges from charged PVC disks and plotted the gap at which discharge has occurred against the disk-charge density for different values of the disk radius. The graphs are shown in Fig. 4.

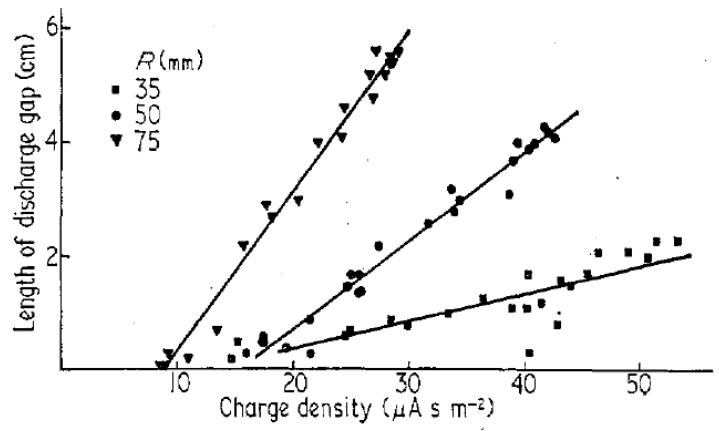

Fig. 4. Length of Discharge Gap as a Function of the Surface Charge Density (Electrode Radius 7.5 mm; Positive Discharges from PVC) (Reproduced from [6])

As these graphs (Fig. 4) show, the length of the discharge gap increases as the disk-charge density increases.

These results give evidence against the validity of equation (3) which appears in Walmsley's calculations [2]. Since this equation results from the relation $\Delta Q_{\mathrm{g}}=\pi r_{\text {eff }}{ }^{2} \sigma$ which is valid and the empirical relation (1), it seems reasonable to suspect the validity of equation (1). Thus, one source of error in Walmsley's model seems to be the use of the empirical relation (1), i.e., the relation $r_{\mathrm{eff}}=K D$.

\section{Probable Solution}

The equation in the paper that we are unsure of is

$$
r_{\mathrm{eff}}=K D
$$

So, we need to find a solution for the charge-collection efficiency $\varepsilon_{\mathrm{m}}$ without using this equation. Now, we have

$$
\begin{gathered}
\Delta Q_{\mathrm{g}}=\pi r_{\mathrm{eff}}^{2} \sigma, \\
\text { or, } \Delta Q_{\mathrm{g}}{ }^{\prime}=r_{\mathrm{eff}}{ }^{2} \sigma^{\prime} \ldots(6),
\end{gathered}
$$

where the primed symbols denote the quantities in their normalized forms [2]. We also have

$$
\begin{gathered}
r_{\mathrm{eff}}^{2}=\alpha_{\mathrm{d}} r_{\mathrm{d}}^{2}, \\
\text { or, } r_{\mathrm{eff}}{ }^{2}=\alpha_{\mathrm{d}} r_{\mathrm{d}}^{2} \ldots(7),
\end{gathered}
$$

where the primed symbols denote the quantities in their normalized forms [2].

The breakdown criterion is given by [2] 


$$
\sigma^{\prime}=\left\{\frac{\left(D^{\prime}-1\right)\left(D^{\prime}-3\right)+R^{\prime 2}}{\sqrt{\left(D^{\prime}-1\right)^{2}+R^{\prime 2}}}-\left(D^{\prime}-3\right)\right\}^{-1}
$$

We also have the equation for the charge-collection efficiency as follows [2]:

$$
\varepsilon_{m}=1-\frac{2}{r_{d}{ }^{\prime 2}}\left[\sqrt{D^{\prime 2}+r_{d}^{\prime 2}}-D^{\prime}\right] \ldots(9) \text {. }
$$

From equations (6) and (7), we have

$$
\Delta Q_{\mathrm{g}}{ }^{\prime}=\alpha_{\mathrm{d}} r_{\mathrm{d}}{ }^{2} \sigma^{\prime} \ldots(10) \text {. }
$$

Now, from equations (8) and (10), we get

$$
\begin{gathered}
\Delta Q_{g}^{\prime}=\alpha_{d} r_{d}^{\prime 2}\left\{\frac{\left(D^{\prime}-1\right)\left(D^{\prime}-3\right)+R^{\prime 2}}{\sqrt{\left(D^{\prime}-1\right)^{2}+R^{\prime 2}}}-\left(D^{\prime}-3\right)\right\}^{-1}, \\
\text { or, } r_{d}^{\prime 2}=\frac{\Delta Q_{g}^{\prime}}{\alpha_{d}}\left\{\frac{\left(D^{\prime}-1\right)\left(D^{\prime}-3\right)+R^{\prime 2}}{\sqrt{\left(D^{\prime}-1\right)^{2}+R^{\prime 2}}}-\left(D^{\prime}-3\right)\right\} \ldots(1)
\end{gathered}
$$

Now, putting this expression for ${r_{\mathrm{d}}}^{2}$ into equation (9), we have

$$
\begin{aligned}
\varepsilon_{m}=1-\frac{2 \alpha_{d}}{\Delta Q_{g}^{\prime}} & \left\{\frac{\left(D^{\prime}-1\right)\left(D^{\prime}-3\right)+R^{\prime 2}}{\sqrt{\left(D^{\prime}-1\right)^{2}+R^{\prime 2}}}-\left(D^{\prime}-3\right)\right\}^{-1} \\
& \times\left[\sqrt{D^{\prime 2}+\frac{\Delta Q_{g}^{\prime}}{\alpha_{d}}\left\{\frac{\left(D^{\prime}-1\right)\left(D^{\prime}-3\right)+R^{\prime 2}}{\sqrt{\left(D^{\prime}-1\right)^{2}+R^{\prime 2}}}-\left(D^{\prime}-3\right)\right\}}-D^{\prime}\right] \ldots
\end{aligned}
$$

Again,

$$
\begin{array}{r}
\varepsilon_{m}=\frac{\Delta Q_{m}^{\prime}}{\Delta Q_{g}^{\prime}}, \\
\text { or, } \Delta Q_{g}^{\prime}=\frac{\Delta Q_{m}^{\prime}}{\varepsilon_{m}} \ldots
\end{array}
$$

where $\Delta Q_{m}^{\prime}$ is the measurement of charge-transfer obtained from the coulombmeter.

Putting this expression of $\Delta Q_{g}^{\prime}$ into equation (12), we have 


$$
\begin{aligned}
\varepsilon_{m}=1-\frac{2 \alpha_{d} \varepsilon_{m}}{\Delta Q_{m}^{\prime}} & \left\{\frac{\left(D^{\prime}-1\right)\left(D^{\prime}-3\right)+R^{\prime 2}}{\sqrt{\left(D^{\prime}-1\right)^{2}+R^{\prime 2}}}-\left(D^{\prime}-3\right)\right\}^{-1} \\
& \times\left[\sqrt{D^{\prime 2}+\frac{\Delta Q_{m}^{\prime}}{\alpha_{d} \varepsilon_{m}}\left\{\frac{\left(D^{\prime}-1\right)\left(D^{\prime}-3\right)+R^{\prime 2}}{\sqrt{\left(D^{\prime}-1\right)^{2}+R^{\prime 2}}}-\left(D^{\prime}-3\right)\right\}}-D^{\prime}\right] \ldots
\end{aligned}
$$

Using equation (14), we may obtain the value of $\varepsilon_{\mathrm{m}}$ for every set of values of $D^{\prime}, R^{\prime}$ and $\Delta Q_{m}{ }^{\prime}$ taking some appropriate value of $\alpha_{\mathrm{d}}$, such as, 0.75, as has been taken in the referred paper. This would possibly be a more reliable method of obtaining the charge-collection efficiency than that used in the referred paper.

\section{Conclusion}

Thus, we find that a possible source of error in Walmsley's calculations is the use of the equation $r_{\text {eff }}=K D$. A more reliable method of calculating the value of the charge-collection efficiency $\varepsilon_{\mathrm{m}}$ would be to use equation (14) and obtain its value for every set of values of $D^{\prime}$, $R^{\prime}$ and $\Delta Q_{m}^{\prime}$, which is the measurement obtained from the coulombmeter.

\section{References}

1. Indian Standard IS/IEC 60079-0:2007, Explosive Atmospheres — Part 0: Equipment — General Requirements, May 2012.

2. H. L. Walmsley, "Induced-charge errors in charge-transfer measurement: Brush discharges between charged, insulating discs and earthed, conductive spheres", Journal of Electrostatics, Vol. 68, 2010, pp. 5 - 20.

3. K. Chowdhury and U. von Pidoll, "Experimental investigation on the measurement deviations in recording brush discharges from insulating surfaces by Coulombmeters", Journal of Electrostatics, Vol. 70, 2012, pp. 347 -355 .

4. E. Heidelberg, "Test and judgment of ignition hazards caused by electrostatic spray guns and charged surfaces without the use of explosive mixtures", Journal of Electrostatics, Vol. 21, 1988, pp. 1 - 17.

5. E. U. Landers, "Distribution of Charge and Fieldstrength Due to Discharge from Insulating Surfaces", Journal of Electrostatics, Vol. 17, 1985, pp. $59-68$.

6. O. Fredholm and K. G. Lövstrand, "On the Discharge of Static Electricity from an Insulator Surface", Journal of Physics E: Scientific Instruments, Vol. 5, 1972, pp. 1058 - 1062. 